C. McMullen

Nagoya Math. J.

Vol. 96 (1984), 1-9

\title{
THE HAUSDORFF DIMENSION OF GENERAL SIERPIŃSKI CARPETS
}

\author{
CURT MCMULLEN
}

\section{§1. Introduction}

In this note we determine the Hausdorff dimension of a family of planar sets which are generalizations of the classical Cantor set. Given $n \geq m$ and a set $R$ consisting of pairs of integers $(i, j)$ with $0 \leq i<n$ and $0 \leq j<m$, define the set $\bar{R}$ by

$$
\bar{R}=\left\{\left(\sum_{i=1}^{\infty} \frac{x_{k}}{n^{k}}, \sum_{1}^{\infty} \frac{y_{k}}{m^{k}}\right):\left(x_{k}, y_{k}\right) \in R \forall k\right\} .
$$

We refer to $\bar{R}$ as a general Sierpinski carpet, after Mandelbrot [4], since Sierpiński's universal curve is a special case of this construction [6].

It is clear that $\bar{R}=\bigcup_{1}^{r} f_{i}(\bar{R})$, where $r=|R|$ and the $f_{i}$ are affine maps contracting $\bar{R}$ by a factor of $n$ horizontally and $m$ vertically. When $n=m$ these maps are actually similarity transformations, and a wellknown argument shows the dimension of $\bar{R}$ is $\log r / \log n$ (following e.g. Beardon [1]). If $n>m$, however, a different approach is required, essentially because squares are stretched into narrow rectangles under iteration of the maps $f_{i}$.

Our method relies on elementary probability theory to address the general case, and we obtain the following result.

TheOREM. The Hausdorff dimension of $\bar{R}$ is given by

$$
\operatorname{dim} \bar{R}=\log _{m}\left(\sum_{j=0}^{m-1} t_{j}^{\left(\log _{n} m\right)}\right)
$$

where $t_{j}$ is the number of $i$ such that $(i, j) \in R$.

This settles a question of Hironaka's [2] concerning the dimension of a certain continuous plane curve whose self-similarities entail the

Received December 27, 1982. 
stretching described above. By the theorem, the dimension of Hironaka's curve is $\log _{2}\left(1+2^{\left(\log _{3} 2\right)}\right)$; his example is essentially the same as the Sierpiński carpet $\bar{R}$ for $m=2, n=3$ and $R=\{(0,0),(1,1),(2,0)\}$. (See Fig. 1.)

The theorem is proved in Section 2. In Section 3 we recall the notion of metric dimension and discuss the following result.

Proposition. The metric dimension of $\bar{R}$ is given by

$$
\mathrm{m} \cdot \operatorname{dim}(\bar{R})=\log _{m} s+\log _{n}(r / s)
$$

where $s$ is the number of $j$ such that $(i, j) \in R$ for some $i$, and $r=|R|$.

The equality of the metric and Hausdorff dimensions of $\bar{R}$ when $n=m$ sheds some light on the distinguished character of that case.

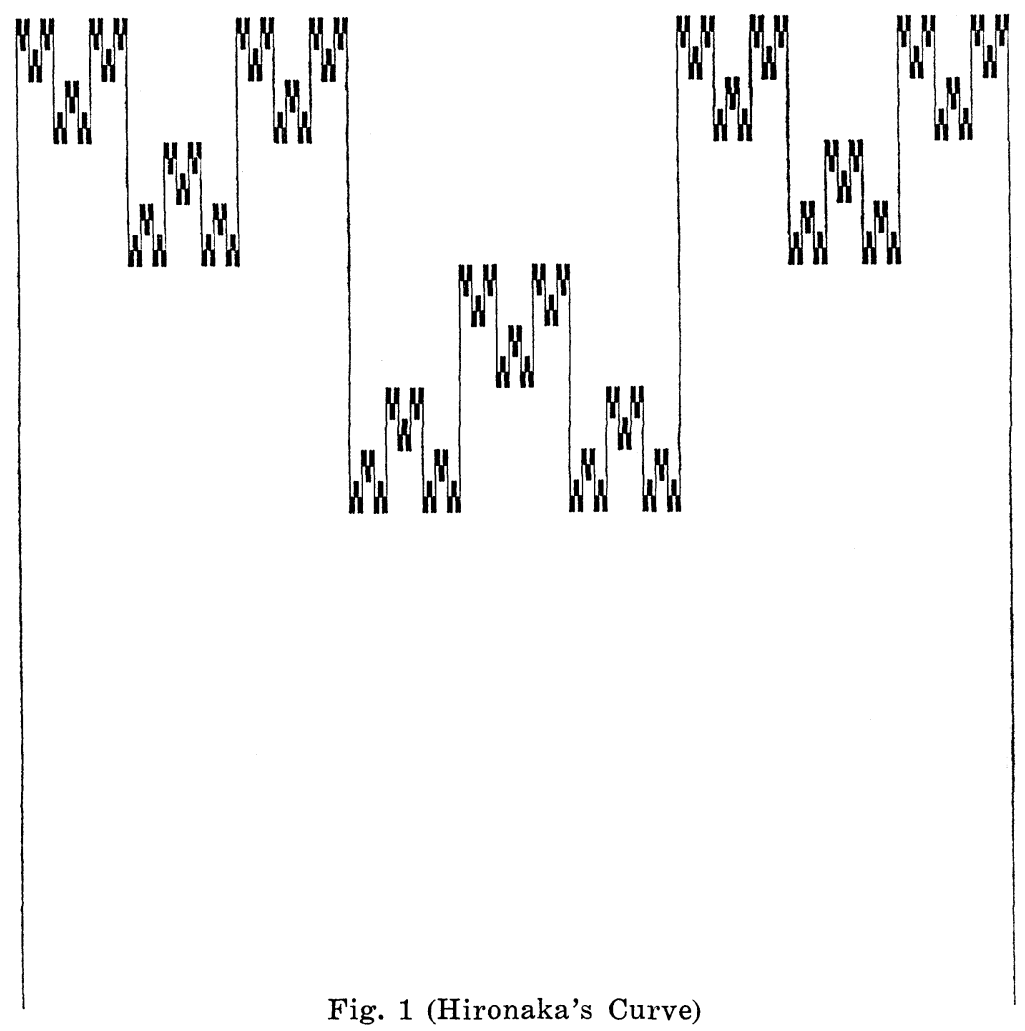




\section{§2. The Hausdorff dimension of $\bar{R}$}

Let $X$ be a metric space. A collection of sets $\left(X_{i}\right)$ is an $\varepsilon$-cover of $X$ if $X=\cup_{1}^{\infty} X_{i}$ and $\operatorname{diam}\left(X_{i}\right)<\varepsilon$ for all $i$. The $\delta$-dimensional Hausdorff measure of $X$ is given by

$$
\mu_{\delta}(X)=\sup _{\varepsilon>0} \inf \left\{\sum \operatorname{diam}\left(X_{i}\right)^{\delta}:\left(X_{i}\right) \text { is an } \varepsilon \text {-cover of } X\right\} .
$$

We define the Hausdorff dimension of $X$ by

$$
\begin{aligned}
\operatorname{dim} X & =\inf \left\{\delta: \mu_{\delta}(X)=0\right\} \\
& =\sup \left\{\delta: \mu_{\delta}(X)=\infty\right\} .
\end{aligned}
$$

The determination of the Hausdorff dimension of $\bar{R}$ is organized into the following steps.

1. We reformulate the dimension of $\bar{R}$ in terms of coverings by a selected class of rectangles.

2. The covering problem is lifted to a sequence space through a $\operatorname{map} \psi: S_{r} \rightarrow \bar{R}$.

3. A probability measure is introduced on $S_{r}$, and we define a sequence of functions $f_{k}$ which measure the difference between this measure and the $\delta$-dimensional Hausdorff measure on $\bar{R}$. Here $\delta$ is the value of $\operatorname{dim} \bar{R}$ claimed in the theorem.

4. We prove a) $\varlimsup$ im $f_{k} \geq 1$ on all of $S_{r}$ and

b) $\lim f_{k}=1$ almost everywhere.

5. We use (a) to show $\operatorname{dim} \bar{R} \leq \delta$.

6. We use (b) to show $\operatorname{dim} \bar{R} \geq \delta$.

Each step corresponds to a lemma below.

First, consider coverings of $\bar{R}$ by rectangles of the form

$$
R_{k}(p, q)=\left[\frac{p}{n^{l}}, \frac{p+1}{n^{l}}\right] \times\left[\frac{q}{m^{k}}, \frac{q+1}{m^{k}}\right]
$$

where $l=\left[k \log _{n} m\right]$ is the integer such that $n>m^{k} / n^{l} \geq 1$. (In the sequel $l$ will always be related to $k$ in this way.) These rectangles are of course tailored to the structure of $\bar{R}$. To a covering $C=\left\{R_{k}(p, q)\right\}$ we associate the integers $N_{k}=$ the number of $R_{k^{\prime}}(p, q) \in C$ with $k^{\prime}=k$.

Lemma 1. The Hausdorff measure $\mu_{\delta}(\bar{R})=0$ iff for any $\varepsilon>0$ there exists a covering $C$ of $\bar{R}$ with $\sum N_{k} m^{-\delta k}<\varepsilon$. 
The proof is straightforward upon observing that (a) any bounded set can be covered by $N$ rectangles $R_{k}(p, q)$ of smaller diameter, where $N$ is a fixed constant; and (b) $m^{-k} \simeq \operatorname{diam} R_{k}(p, q)$.

Now let $\left(x_{i}, y_{i}\right)_{i=0}^{r-1}$ enumerate the elements of $R$, and let $S_{r}=$ $\prod_{1}^{\infty}\{0,1,2, \cdots, r-1\}$. Then the map $\psi: S_{r} \rightarrow \bar{R}$ given by

$$
\Psi:\left(i_{1}, i_{2}, i_{3}, \cdots\right) \longrightarrow\left(\sum \frac{x_{i_{k}}}{n^{k}}, \sum \frac{y_{i_{k}}}{m^{k}}\right)
$$

is a surjection. (It fails to be injective because some coordinates have more than one "decimal" representation.) We will construct sets $A_{k}(p, q)$ $\times B_{k}$ in $S_{r}$ which correspond roughly to the sets $R_{k}(p, q)$ in $\bar{R}$ under the map $\psi$. Writing

$$
p=\sum_{1}^{l} \tilde{x}_{j} n^{l-j} \quad 0 \leq \tilde{x}_{j}<n, l=\left[k \log _{n} m\right]
$$

and

$$
q=\sum_{1}^{k} \tilde{y}_{j} m^{k-j} \quad 0 \leq \tilde{y}_{j}<m
$$

we define

$$
\begin{aligned}
A_{k}(p, q)=\left\{\left(i_{1}, i_{2}, \cdots, i_{k}\right): x_{i_{j}}=\right. & \tilde{x}_{j} \text { for } j=1, \cdots, l \\
& \text { and } \left.y_{i_{j}}=\tilde{y}_{j} \text { for } j=1, \cdots, k\right\}
\end{aligned}
$$

and

$$
B_{k}=\prod_{k+1}^{\infty}\{0,1, \cdots, r-1\} .
$$

To a covering $C=\left\{A_{k}(p, q) \times B_{k}\right\}$ we also associate the integers $N_{k}=$ the number of $A_{k^{\prime}}(p, q) \times B_{k^{\prime}} \in C$ with $k^{\prime}=k$.

Lemma 2. The Hausdorff measure $\mu_{\delta}(\bar{R})=0$ iff for any $\varepsilon>0$, there exists a covering $C$ of $S_{r}$ with $\sum N_{k} m^{-\delta k}<\varepsilon$.

Proof. Note that

$$
A_{k}(p, q) \times B_{k} \subset \Psi^{-1}\left(R_{k}(p, q)\right) \subset \underset{\substack{i=-1,0,1 \\ j=-1,0,1}}{\bigcup} A_{k}(p+i, q+j) \times B_{k}
$$

so we can pass back and forth between covers of $\bar{R}$ and covers of $S_{r}$. Furthermore we need only change $N_{k}$ by a bounded factor in doing so. The result then follows from Lemma 1.

The point of lifting the problem to $S_{r}$ is that coverings of a product space by cylindrical sets are more easily analyzed than coverings of $\bar{R}$ by rectangles. 
We now study the size of the cylinders $A_{k}(p, q) \times B_{k}$. For $i=$ $0,1, \cdots, r-1$, let $a_{i}=$ the number of $j$ such that $y_{i}=y_{j}$, where $\left(x_{i}, y_{i}\right)_{i=0}^{r-1}$ enumerates $R$ as before. Then the cardinality of $A_{k}(p, q)$ is given by the product $a_{i_{l+1}} a_{i_{l_{+2}}} \cdots a_{i_{k}}$, for any $\left(i_{1}, i_{2}, \cdots, i_{k}\right) \in A_{k}(p, q)$.

The constants $a_{i}$ will be used to define a probability measure $\mu$ on $S_{r}$. First, let $\delta$ be the value of $\operatorname{dim} \bar{R}$ claimed in the statement of the theorem. Note that

$$
m^{\delta}=\sum_{0}^{r-1} a_{i}^{\left(\log _{n} m-1\right)} .
$$

Letting $b_{i}=a_{i}^{\left(\log _{n} m-1\right)} / m^{\delta}$, we have $\sum b_{i}=1$. We take $\mu$ to be the unique probability measure on the Borel subsets of $S_{r}$ (in the product topology) which satisfies, for any $\left(i_{1}, i_{2}, \cdots, i_{k}\right)$,

$$
\mu\left(\left(i_{1}, i_{2}, \cdots, i_{k}\right) \times B_{k}\right)=b_{i_{1}} b_{i_{2}} b_{i_{3}} \cdots b_{i_{k}} .
$$

The existence of such a measure is a special case of the Kolmogorov consistency theorem (see [5]).

Define the functions $f_{k}$ on $S_{r}$ by

$$
f_{k}\left(i_{1}, i_{2}, i_{3}, \cdots\right)=\left[\frac{\left(a_{i_{1}} a_{i_{2}} \cdots a_{i_{k}}\right)^{\log _{n} m}}{\left(a_{i_{1}} a_{i_{2}} \cdots a_{i_{l}}\right)}\right]^{1 / k}
$$

where $l=\left[k \log _{n} m\right]$ as usual.

Lemma 3. If $z \in A_{k}(p, q) \times B_{k}$, then

$$
\mu\left(A_{k}(p, q) \times B_{k}\right)=\left(f_{k}(z) m^{-\delta}\right)^{k} .
$$

Proof. Pick $\left(i_{1}, i_{2}, \cdots, i_{k}\right) \in A_{k}(p, q)$ and define the sequence

$$
\left(s_{1}, s_{2}, \cdots, s_{k}\right)=\left(a_{i_{1}}, a_{i_{2}}, \cdots, a_{i_{k}}\right) .
$$

Note that this sequence is independent of the choice of $\left(i_{1}, i_{2}, \cdots, i_{k}\right)$. In particular,

$$
\mu\left(\left(i_{1}, i_{2}, \cdots, i_{k}\right) \times B_{k}\right)=\frac{\left(s_{1} s_{2} \cdots s_{k}\right)^{\log _{n} m}}{\left(s_{1} s_{2} \cdots s_{k}\right)} m^{-\delta k}
$$

for each $\left(i_{1}, i_{2}, \cdots, i_{k}\right) \in A_{k}(p, q)$. But the cardinality of $A_{k}(p, q)$ is $s_{l+1} s_{l+2} \cdots s_{k}$, so

$$
\mu\left(A_{k}(p, q) \times B_{k}\right)=\frac{\left(s_{1} s_{2} \cdots s_{k}\right)^{\log _{n} m}}{\left(s_{1} s_{2} \cdots s_{l}\right)} m^{-\delta k}=\left(f_{k}(z) m^{-\delta}\right)^{k}
$$

as claimed. 
It remains only to show that the functions $f_{k} \rightarrow 1$ in a suitable sense, so that for a covering $C$ of $S_{r}$ we have

$$
\sum N_{k} m^{-\delta k} \approx \sum \mu\left(A_{k}(p, q) \times B_{k}\right)
$$

and we can apply Lemma 2.

LEMMA 4. a) $\varlimsup_{\lim } f_{k}(z) \geq 1$ for all $z \in S_{r}$.

b) $f_{k} \rightarrow 1$ almost everywhere $(\mu)$.

Proof. Define $g_{k}, h_{k}$ on $S_{r}$ by

$$
\begin{aligned}
& g_{k}\left(i_{1}, i_{2}, \cdots\right)=\frac{\left(a_{i_{1}} a_{i_{2}} \cdots a_{i_{k}}\right)^{1 / k}}{\left(a_{i_{1}} a_{i_{2}} \cdots a_{i_{l}}\right)^{1 / l}} \\
& h_{k}\left(i_{1}, i_{2}, \cdots\right)=\left[a_{i_{1}} a_{i_{2}} \cdots a_{i_{l}}\right]^{1 / l)\left(\log _{n} m-l / k\right)} .
\end{aligned}
$$

Then $f_{k}(z)=h_{k}(z) \cdot g_{k}(z)^{\log _{n} m}$. We claim $h_{k}(z) \rightarrow 1$ for all $z$. Indeed, $1 \leq$ $a_{i} \leq n$ for all $i$, so

$$
1 \leq h_{k} \leq n^{\left(\log _{n} m-l / k\right)}
$$

and

$$
\log _{n} m-\frac{l}{k}=\log _{n} m-\frac{\left[k \log _{n} m\right]}{k} \longrightarrow 0 \quad \text { as } k \longrightarrow \infty
$$

Therefore it suffices to show a) $\varlimsup_{k} g_{k}(z) \geq 1$ for all $z \in S_{r}$ and b) $g_{k}(z) \rightarrow 1$ a.e.

(a) Fix $z=\left(i_{1}, i_{2}, \cdots\right)$ and let $s_{j}=\left(a_{i_{1}} a_{i_{2}} \cdots a_{i_{j}}\right)^{1 / j}$. Then $1 \leq s_{j}$, so it is clear that

$$
\varlimsup g_{k}(z)=\varlimsup \frac{s_{k}}{s_{l}} \geq 1
$$

since this holds for any positive sequence $s_{j}$ bounded away from zero.

(b) From the definition of $\mu$ it is almost tautological to assert that the functions

$$
\left(i_{1}, i_{2}, \cdots\right) \longrightarrow b_{i_{k}} \quad k=1,2,3, \cdots
$$

are independent, identically distributed random variables with respect to $\mu$. Hence the sequence

$$
\left(b_{i_{1}} b_{i_{2}} \cdots b_{i_{k}}\right)^{1 / k} \quad k=1,2,3, \cdots
$$

converges for almost every $\left(i_{1}, i_{2}, \cdots\right) \in S_{r}$, by Kolmogorov's strong law of large numbers [3]. Recalling the definition of $b_{i}$ we have 


$$
g_{k}\left(i_{1}, i_{2}, \cdots\right)=\left[\frac{\left(b_{i_{1}} b_{i_{2}} \cdots b_{i_{k}}\right)^{1 / k}}{\left(b_{i_{1}} b_{i_{2}} \cdots b_{i_{l}}\right)^{1 / l}}\right]^{\left(\log _{n} m-1\right)-1}
$$

and hence $g_{k} \rightarrow 1$ almost everywhere. (In case $n=m$ we have $l=k$ so $g_{k}$ is identically 1 ).

Lemma $5 . \operatorname{dim} \bar{R} \leq \delta$.

Proof. We will construct an efficient cover for $S_{r}$.

Fix $\varepsilon>0$.

Let $C_{k}$ consist of those nonempty sets $A_{k}(p, q) \times B_{k}$ for which $f_{k}(z)$ $>m^{-\varepsilon}$ for $z \in A_{k}(p, q) \times B_{k}$. These sets are disjoint and satisfy

$$
\mu\left(A_{k}(p, q) \times B_{k}\right)>m^{-(\delta+\varepsilon) k}
$$

by Lemma 3. Hence $M_{k}=$ cardinality of $C_{k}<m^{(\tilde{o}+\varepsilon) k}$ as the measure of $S_{r}$ is one.

Note that any $z \in S_{r}$ is covered by $C_{k}$ for infinitely many $k$, since $\varlimsup \lim f_{k}(z) \geq 1>m^{-\varepsilon}$. Hence $C=\bigcup_{k \geq K} C_{k}$ is a covering of $S_{r}$ for any choice of $K$. Choose $K$ large enough that $\sum_{k \geq K} m^{-\varepsilon k}<\varepsilon$; then the $N_{k}$ associated to $C$ satisfy

$$
\sum N_{k} m^{-(\delta+2 \varepsilon) k}=\sum_{k \geq K} M_{k} m^{-(\delta+2 \varepsilon)}<\sum_{k \geq K} m^{-\varepsilon k}<\varepsilon .
$$

Using Lemma 2 it then follows easily that $\operatorname{dim} \bar{R} \leq \delta$.

LEMMA $6 . \operatorname{dim} \bar{R} \geq \delta$.

Proof. Fix $\beta<\delta$. We will show there exists an $\varepsilon>0$ such that $\sum N_{k} m^{-\beta k}>\varepsilon$ for any covering $C$ of $S_{r}$; the dimension estimate is then immediate from Lemma 2.

Let $E_{K}=\left\{z \in S_{r}: f_{k}(z)<m^{\delta-\beta}\right.$ for all $\left.k \geq K\right\}$. We know $f_{k}$ tends to 1 almost everywhere, and $m^{\delta-\beta}>1$, so we can pick $K$ such that $\mu E_{K}>0$. Set $\varepsilon=\min \left\{\mu E_{K}, m^{-\beta K}\right\}$.

Now let $C$ be any covering of $S_{r}$. If $N_{k} \neq 0$ for some $k<K$, then $\sum N_{k} m^{-\beta k}>m^{-\beta K}>\varepsilon$. So assume that $N_{k}=0$ for $k<K$.

Then for the elements of $C$ such that $A_{k}(p, q) \times B_{k} \cap E_{K} \neq \phi$, we have

$$
\mu\left(A_{k}(p, q) \times B_{k}\right)=\left[f_{k}(z) m^{-\delta}\right]^{k}<\left[m^{\delta-\beta} \cdot m^{-\delta}\right]^{k}=m^{-\beta k}
$$

where we have chosen $z$ lying the intersection of the two sets, and used the assumption that $k \geq K$. Since $C$ covers $E_{K}$, we have $\sum N_{k} m^{-\beta k}>$ 
$\mu E_{K} \geq \varepsilon$. By Lemma $2, \mu_{\beta}(\bar{R}) \neq 0$; since $\beta<\delta$ was arbitrary, it follows that $\operatorname{dim}(\bar{R}) \geq \delta$.

The preceding two lemmas complete the proof of the theorem.

\section{$\S 3$. The metric dimension of $\bar{R}$}

Let $X$ be a bounded finite dimensional metric space, and let $N(\varepsilon)$ denote the number of elements in a minimal covering of $X$ by $\varepsilon$-balls. The metric dimension of $X$ is given by

$$
\operatorname{m.dim}(X)=\varlimsup_{\varepsilon \rightarrow 0} \frac{\log N(\varepsilon)}{\log 1 / \varepsilon} .
$$

The metric dimension can be thought of as the value obtained for the Hausdorff dimension if we restrict ourselves to coverings all of whose elements have the same size. Consequently the inequality $m \cdot \operatorname{dim}(X) \geq$ $\operatorname{dim}(X)$ always holds.

Now let $N_{k}$ denote the number of $(p, q)$ such that $R_{k}(p, q) \cap \bar{R} \neq \phi$. It is easy to see there exist $C_{1}, C_{2}>0$ such that $C_{1} N_{k}>N\left(m^{-k}\right)>C_{2} N_{k}$, and therefore m.dim $(\bar{R})=\varlimsup \lim \log N_{k} / \log m^{k}$. But $N_{k}$ is precisely the number of ways to choose $\left(x_{i}\right)_{i=1}^{l}$ and $\left(y_{i}\right)_{i=1}^{k}$ such that

a) $\left(x_{i}, y_{i}\right) \in R$ for $i=1,2, \cdots, l$, and

b) $\left(\tilde{x}_{i}, y_{i}\right) \in R$ for $i=l+1, l+2, \cdots, k$ and some choice of $\tilde{x}_{i}$.

Hence $N_{k}=r^{l} s^{k-l}=(r / s)^{l} s^{k}$ where $r$ is the cardinality of $R$ and $s$ is the number of $y$ such that $(x, y) \in R$ for some $x$; and the metric dimension of $\bar{R}$ is given by

$$
\begin{aligned}
\mathrm{m} \cdot \operatorname{dim}(\bar{R}) & =\varlimsup_{k} \frac{\log (r / s)^{1} s^{k}}{\log m^{k}}=\log _{m} s+\log _{m}\left(\frac{r}{s}\right) \varlimsup \lim \left(\frac{1}{k}\right) \\
& =\log _{m} s+\log _{m}\left(\frac{r}{s}\right) \log _{n} m=\log _{m} s+\log _{n}\left(\frac{r}{s}\right)
\end{aligned}
$$

as stated in the introduction.

There are two conditions under which the Hausdorff and metric dimensions of $\bar{R}$ agree. The first is when $n=m$ : in this case the set $\bar{R}$ is geometrically self-similar and both dimensions are given by $\log _{n} r$ as noted in the introduction. The second condition occurs when the constants $t_{j}$ take on only one value other than zero. (Recall $t_{j}$ is the number of $i$ such that $(i, j) \in R)$.

As an example of the latter case, suppose $R=T \times U$. Then 


$$
\operatorname{dim}(\bar{R})=\log _{m}\left(u t^{\left(\log _{n} m\right)}\right)=\log _{m} u+\log _{n} t=\mathrm{m} \cdot \operatorname{dim}(\bar{R})
$$

where $u=|U|$ and $t=|T|$. Indeed, $\bar{R}=\bar{T} \times \bar{U}$ where $\bar{T}$ and $\bar{U}$ are general Cantor sets in $R$, and we are just asserting that

$$
\operatorname{dim}(\bar{R})=\operatorname{m} \cdot \operatorname{dim}(\bar{R})=\operatorname{dim}(\bar{T})+\operatorname{dim}(\bar{U}) .
$$

This also follows from a general theorem due to Wegmann [7] which states that $\operatorname{dim}\left(E_{1} \times E_{2}\right)=\operatorname{dim}\left(E_{1}\right)+\operatorname{dim}\left(E_{2}\right)$ whenever m.dim $\left(E_{i}\right)=$ $\operatorname{dim}\left(E_{i}\right)$ for $i=1,2$.

We remark that the functions $f_{k}$ introduced in the preceding section are identically equal to one if the Hausdorff and metric dimensions of $\bar{R}$ agree, and the proof can be simplified in this case. Furthermore one easily obtains the stronger statement that the Hausdorff measure of $\bar{R}$ satisfies $0<\mu_{\delta} \bar{R}<\infty$. It would be interesting to know if this last assertion holds in general.

\section{REFERENCES}

[1] A. F. Beardon, On the Hausdorff dimension of general Cantor sets, Proc. Camb. Phil. Soc., 61 (1965), 679-694.

[2] H. Hironaka, Hironaka Heisuke no Suugaku Kyositsu, Tokyo 1980.

[ 3 ] Michael Loeve, Probability Theory, New York 1963.

[4] Benoit B. Mandelbrot, Fractals, San Francisco, 1977.

[5] K. R. Parthasarathy, Probability Measures on Metric Spaces, New York, 1967.

[6] W. Sierpiński, "Sur une courbe cantorienne qui contient une image biunivoque et continue de toute courbe donné", Comptes Rendus, 162 (1916), 629-642.

[7] H. Wegmann, "Die Hausdorff-Dimension von kartesischen Produkten metrischer Räume”, J. reine angew. Math., 246 (1971), 46-75.

Department of Mathematics

Harvard University

Cambridge Massachusetts 01238

USA 